\title{
Aprendizaje en línea en la EsaCE, una experiencia enriquecedora en tiempos de pandemia ${ }^{1}$
}

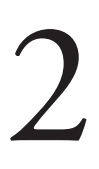

https://doi.org/10.21830.9789585350663.02

\author{
Heidi Isabel Merlano Niebles ${ }^{2}$ \\ Joana Patricia Medina ${ }^{3}$ \\ Escuela de Armas Combinadas del Ejército
}

Resumen. Este artículo presenta los resultados de una investigación con método mixto realizada durante el confinamiento obligatorio en Colombia como consecuencia de la Covid-19. Específicamente, se analizan las dimensiones organizativas, pedagógicas, tecnológicas, gestión, diseño, interfaz y orientación respecto a la satisfacción y experiencia de los militares que realizaron los cursos de ascenso durante el año 2020 y cuya capacitación fue en línea por la pandemia. Los resultados cuantitativos muestran que estos estudiantes aprueban el modelo de aprendizaje, el cual les ha brindado una mejor opción en calidad y tiempo para compartir en familia. Asimismo, los resultados cualitativos evidencian la necesidad de mejorar aspectos como los usos de plataforma y su servicio, así como en las competencias previas en determinados saberes por parte de los alumnos. Para ello se hace necesario prestar atención a aspectos como: comprensión lectora, conocimientos en ofimática e internet, didáctica, atención oportuna y actitud.

Palabras clave: aprendizaje en línea; competencia educativa; dimensión pedagógica; dimensión tecnológica; informática educativa; curso de ascenso militar.

1 Este capítulo forma parte de los resultados del proyecto de investigación "Experiencias de aprendizaje virtual en la EsACE, en tiempos de pandemia”, del Grupo de Investigación en Capacitación Militar (Gicam), de la Escuela de Armas Combinadas del Ejército (EsACE), registrado con el código COL0160714 de Minciencias. Los puntos de vista y los resultados de este capítulo pertenecen a las autoras y no reflejan necesariamente los de la EsACE o el Ejército Nacional de Colombia.

2 Comunicadora Social-Periodista. Especialista de Pedagogía y Docencia de la Fundación Universitaria del Área Andina. Magíster en Comunicación e Identidad Corporativa de la Universidad Internacional de La Rioja. ORCID: https://orcid.org/0000-0001-9601-9467 - Contacto: heidimerlanoniebles@ cedoc.edu.co

3 Licenciada en Lenguas Modernas de la Universidad de Cundinamarca. Magíster en Desarrollo Educativo y Social de la Universidad Pedagógica de Bogotá. ORCID: https://orcid.org/0000-0003-21463517 - Contacto: joanamedina@cedoc.edu.co 


\section{Introducción}

El sector educativo, desde hace ya varios años, ha incluido en sus prácticas el uso de Tecnologías de la Información y la Comunicación (Tic), para lo cual ha generado entornos y actividades de aprendizaje que se amoldan a los estilos de vida y a las situaciones cotidianas y adversas que afrontan las personas. En este sentido, las Tic ofrecen una gran variedad de opciones a las entidades educativas por cuanto proporcionan oportunidades educativas a todo nivel para sus potenciales estudiantes, así como a otras personas que deseen capacitarse a través de ellas.

La internet ha apoyado esta forma educativa y ha optimizado recursos para que tanto docentes como estudiantes, a través de un dispositivo electrónico que recibe y procesa datos, puedan simular un ambiente tradicional de estudios en línea, aprovechando la tecnología, la didáctica y los conocimientos sobre el aprendizaje. Para muchos investigadores esta educación en línea debe tener un contenido innovador que engrane en el desarrollo del aprendizaje, independientemente del lugar donde el estudiante se encuentre realizando este modelo educativo. Es decir, es toda una experiencia. Por ello Cotton (citado por Gómez et al., 2005) afirma que "el aprendizaje es un proceso de adquisición de un nuevo conocimiento y habilidad; es decir, puede definirse como un cambio relativamente permanente en el comportamiento o en el posible comportamiento, fruto de la experiencia” (p. 20).

La educación en línea implica contar con acceso a la conectividad de manera igualitaria, lo que ha sido un obstáculo en el camino hacia la educación en línea. Más aún en países subdesarrollados como el nuestro, en donde la preparación de recursos humanos y el desarrollo en temas de infraestructura para utilizar de manera eficiente la tecnología están atados al desarrollo competitivo en el contexto mundial y, por ende, al avance tecnológico.

Esto ha sido muy evidente en Colombia, especialmente en esta época de pandemia, pues ante la necesidad de estar comunicados a través de canales y medios electrónicos se ha evidenciado, notablemente, la falta de cobertura y de accesibilidad a estos por parte de los habitantes, perjudicando especialmente a estudiantes y trabajadores. El gobierno ha sido consciente de ello, a tal punto que el Ministerio de Tecnologías de la Información y Comunicaciones (Mintic) reportó que alrededor de veinte millones de colombianos no tienen 
conexión a banda ancha (Caracol Radio, 2020). Por ello, se ha iniciado un plan de acción para mejorar dicha situación a futuro, sin dejar de avanzar, en estos momentos de pandemia, con las actividades desde casa - como, por ejemplo, las formativas y educativas_- teniendo en cuenta que el proceso de aprendizaje requiere de estrategias y tareas que deben realizar los estudiantes y los docentes.

La decisión de continuar con las actividades educativas programadas para el 2020 ha sido no solo un tema de interés y de relevancia para el gobierno colombiano, sino también para las entidades o centros educativos públicos y privados. Así, el aprendizaje a través de la virtualidad, y en momentos de pandemia, ha obligado a cambiar la visión de la educación en ciertos lugares donde no había sido considerado como una opción principal o alterna para ampliar, complementar o instituirlo como fundamento educativo, ya fuese de manera alterna o constante.

La Escuela de Armas Combinadas del Ejército (EsAce) fue uno de los centros educativos que acogió la medida y continuó con su programación académica. Con esto, la Escuela demostró que cuenta con la capacidad para brindar a su comunidad una oferta educativa en línea — pues los estudiantes militares y los docentes estaban capacitados para afrontar el reto-y que está preparada para esta modalidad de estudios, la cual, sin lugar a dudas, es una prioridad actualmente.

El reto inició con una capacitación por parte de la EsACE a los docentes civiles y militares a través de un seminario en "Formación virtual y nuevas tecnologías en educación 2020", que sirvió como refuerzo de la labor y como una oportunidad para incluir parámetros y directrices relacionados con el ejercicio de aprendizaje virtual y en línea.

La aplicación escogida fue la plataforma Blackboard, a través de la cual los docentes y los estudiantes pudieron crear comunidades y compartir de manera sincrónica y asincrónica procesos de enseńanza-aprendizaje y uso compartido de conocimientos en línea.

La colaboración entre docentes y estudiantes fue la característica principal, tanto en el ámbito institucional como en el individual. Esto se vio reflejado en la conectividad. Muchos utilizaron sus propios planes de internet, banda ancha, wifi, el acceso gratuito que ofrecía el Ejército Nacional a través de sus guarniciones o del Estado a través de su programa Conectividad Total. 
Esta suma de esfuerzos hizo realidad que cientos de militares programados en el 2020 para realizar sus cursos de ascenso no vieran truncada su opción de estudios, pudieran continuar con su carrera militar y optar a un nuevo grado.

En consideración a lo anterior, la medición de las acciones y los logros fue imprescindible para verificar el esfuerzo hecho y la calidad educativa y pedagógica ofertada y realizada (Castellanos, 2014). A este respecto, no solamente con el cumplimiento de parámetros físicos, tecnológicos e institucionales se da el cumplimiento de ciertos criterios, sino que también se debe tomar en cuenta la satisfacción de los estudiantes y su experiencia, ya que ellos constituyen el elemento central en el paradigma de aprendizaje centrado en el estudiante y son uno de los ejes articuladores de la educación virtual (Blanchard \& Muzas, 2016).

En este contexto, la presente investigación analiza cuál ha sido la experiencia de aprendizaje de los militares que, en medio de la pandemia y el confinamiento nacional, están realizando sus cursos de ascenso de manera virtual y en línea en la EsACE. Para ello, se analizaron las dimensiones: organizativa, pedagógica, tecnológica, de diseño de interfaz, de gestión y de orientación, con el enfoque de métodos mixtos. El estudio tiene dos componentes: uno cuantitativo, que analiza los datos obtenidos a través de una encuesta aplicada a los alumnos, y otro cualitativo, obtenido a través de preguntas abiertas, diálogos y entrevistas a estudiantes y varios profesores de la institución educativa militar, así como la propia experiencia de las autoras como docentes en la EsACE.

\section{Marco teórico}

Por su importancia y complejidad, la evaluación del aprendizaje, especialmente la de los estudiantes, es una muestra de conciencia ética y de calidad en temas concernientes a lo conceptual, metodológico, necesidades del proceso pedagógico, al igual que sus causas y consecuencias.

De este modo, además de evaluar el componente estructural, también se analiza el desarrollo del estudiante en correspondencia con las regularidades esenciales del proceso de formación del individuo y con las finalidades sociales que asignan dicha formación en nuestra sociedad (González, 2000).

Con la aplicación de una evaluación del aprendizaje se obtiene como resultado la medición de los límites de la enseñanza, para incorporar ideas que 
amplíen la comprensión del proceso, teniendo en cuenta al estudiante como factor determinante del éxito.

La evaluación es una actividad humana que se aplica en todos los campos; la educación, específicamente la virtual, no está exenta de este proceder. El aumento de ofertas y demanda de esta opción formativa y de la inscripción de estudiantes en esta se convierte en un requerimiento de existencia dinámico como proceso evaluativo, que debe realizarse de manera continua y constante, que abarque a todos los actores y procesos implicados (Casanova, 1992).

Evaluar es asignar un valor a algo, juzgar. Entre las muchas definiciones que existen de evaluación se resalta aquí la fórmula Tenbrink, que considera a la evaluación como "el proceso de obtención de información y de su uso para formular juicios que a su vez se utilizarán para tomar decisiones" (Tenbrink, 2006, p. 19).

Debido a lo anterior, surge la necesidad, como investigación empírica por parte de las autoras, de conocer los factores determinantes, a favor o en contra, del proceso de aprendizaje realizado en la EsACE de manera totalmente virtual. Por tal razón, este capítulo muestra la percepción de los estudiantes militares que en el año 2020 tuvieron que realizar sus cursos de ascenso de manera totalmente en línea, ante el confinamiento obligatorio que determinó el gobierno nacional por la pandemia de la Covid-19.

La investigación crea un modelo de análisis evaluativo conformado por las dimensiones: organizativa, pedagógica, tecnológica, de diseño de interfaz, gestión y de orientación, teniendo como referente lo expuesto en la investigación preliminar realizada por Juan Manuel Bournissen sobre el "Modelo pedagógico virtual", que se transformó luego en tesis doctoral para la Universidad Adventista del Plata (Bournissen, 2014). A continuación, se describen las dimensiones analizadas en este capítulo, con el fin de referenciar y comprender los análisis de métodos que se presentan más adelante.

La dimensión organizativa se refiere tanto a la organización económica como financiera del centro educativo, que es ajena al estudiante. Esta engloba todos los aspectos que puedan ofrecer las entidades para el proceso de enseñanza-aprendizaje de manera coordinada y capaz de adaptarse a las necesidades y requerimientos de los alumnos. Es necesario aclarar que, aunque la educación sea virtual o en línea, las instituciones deben tener todos los componentes que caracterizan a una institución presencial. Así lo expone Juan Carlos 
Tedesco en su "Modelo de sistema educativo en América Latina", avalado por la Organización de las Naciones Unidas para la Educación, la Ciencia y la Cultura (Unesco).

Por otra parte, la dimensión pedagógica mostrará las propiedades del proceso de enseńanza-aprendizaje adaptado a las Tic, de acuerdo con la institución educativa que la establezca, teniendo en cuenta la metodología, el sistema de comunicación y el material didáctico que se utiliza.

La dimensión tecnológica trabaja en conjunto con las experiencias obtenidas de la parte organizativa y de la pedagógica, usando como variable específica el conocimiento tecnológico de los usuarios (estudiantes y docentes). También integra la infraestructura de red, el hardware y el software necesarios para realizar un curso en línea. Este componente está asociado también al diseño de interfaz del curso, a través de las variables de plataforma y de su uso.

$\mathrm{Al}$ conjunto de servicios que reciben los estudiantes de manera previa y durante el curso se denominará dimensión de gestión, la cual nutre a la dimensión de orientación, que incluye el apoyo en el proceso de seguimiento, la motivación y el asesoramiento a los estudiantes antes y durante su proceso de aprendizaje. En esta última son fundamentales la actitud y aptitud del docente, quien siempre tendrá un papel orientador frente a los participantes de su clase.

Con estas dimensiones se complementaría un modelo pedagógico ideal para la educación virtual y en línea, el cual ya ha sido propuesto por Jesús Salinas, Francisca Negre Bennasar e Isabel Torrandell Serra (2005) como didáctica multimedial para la educación superior. Estos investigadores seleccionaron este modelo luego de analizar los modelos creados por más de trescientas universidades en todo el mundo (estudio en el cual se tuvo en cuenta a Colombia y sus universidades: Javeriana, San Buenaventura, de la Amazonia y Escuela Superior de Administración Pública Virtual).

El modelo fue seleccionado debido a que presenta un conjunto completo de elementos que representan la mayoría de las actividades, por no decir todas, que se realizan en una institución que tiene programas a distancia. Por lo tanto, representan una excelente guía para aquellas instituciones que están buscando implementar un modelo por primera vez. (Bournissen, 2014)

Para elegir ese modelo pedagógico, los elementos y su funcionamiento fueron más relevantes que la cantidad de dimensiones (figura 1). Años atrás, 
Talavera (2011) y Khan (2001) plantearon ocho dimensiones esenciales relacionadas con los aspectos institucionales, pedagógicos, tecnológicos, sobre la evaluación, de gestión, recursos y servicios de soporte, éticos y diseño de interfaces. Así, la relevancia de los elementos y su funcionamiento facilita la realización de análisis de ventajas, desventajas y recomendaciones para el ejercicio de enseñanza-aprendizaje entre quienes participan de este.

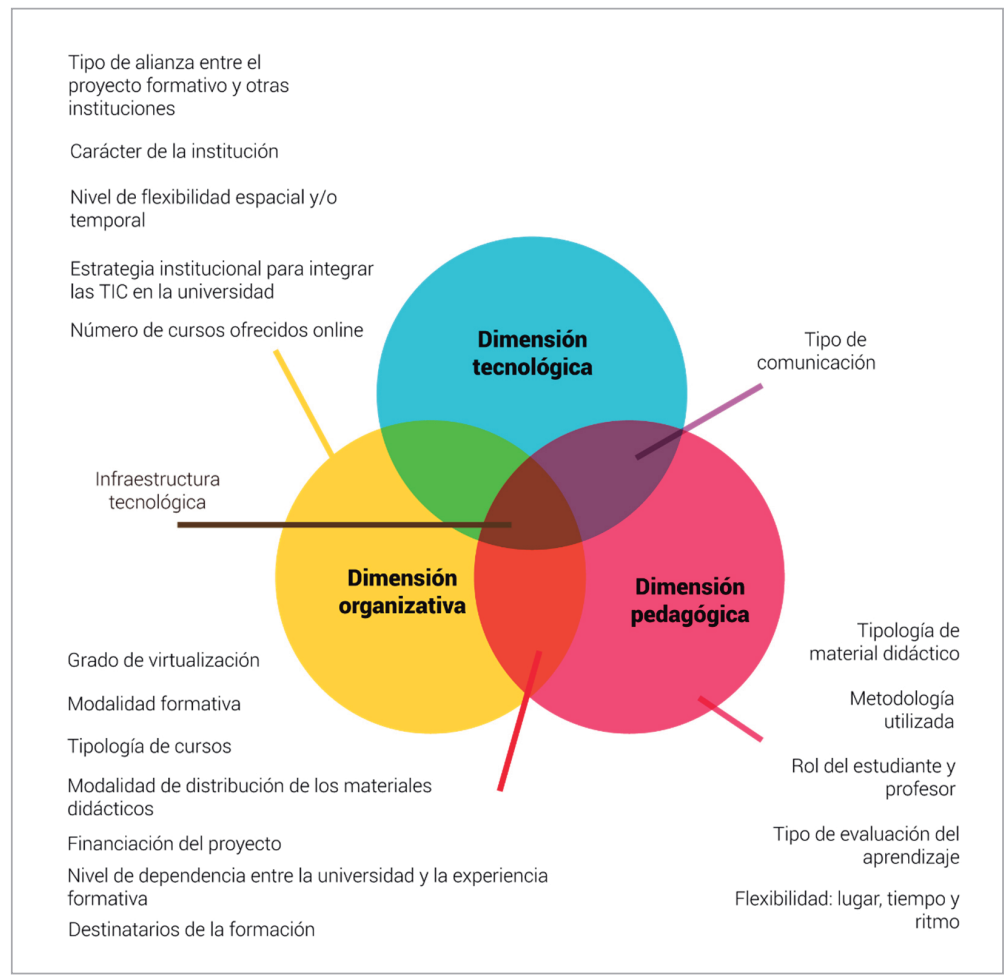

Figura 1. Modelo pedagógico de tres dimensiones.

Fuente: elaboración propia con base en la información recolectada en Bournissen (2014, p. 59).

\section{Métodos}

El presente trabajo forma parte de una investigación con enfoque mixto, corte transversal y alcance descriptivo y explicativo, que tiene por objetivo determinar el nivel de satisfacción y experiencia de los estudiantes militares de la Esace, teniendo en cuenta las diferentes dimensiones y criterios evaluativos, tanto cuantitativos como cualitativos, para determinar correlaciones y 
disconformidades entre los actores que participan en este proceso de enseñanza-aprendizaje.

\section{Contexto y participantes}

La investigación se realizó en la EsACE, institución que funciona desde 1985 brindando capacitación integral a oficiales y suboficiales que ascenderán al grado inmediatamente superior. A la fecha son 35 años consolidándose como la unidad de educación militar fundamental del Ejército Nacional (EsACE, s. f.).

Los oficiales y suboficiales conciben los conceptos dados como un apoyo en su proceso formativo, y como parte de la exigencia de la EsACE y de su carrera militar en el Ejército de Colombia. Así, la EsACE tiene la siguiente misión:

Capacitar integralmente a los Oficiales Subalternos y Suboficiales del Ejército Nacional en las aéreas Táctica, Técnica, Humanística y Administrativa para enfrentar con efectividad las responsabilidades que el cargo y la jerarquía militar exigen; así mismo, apoyar los procesos de generación, actualización y difusión de la doctrina de nivel táctico para la formación de líderes legítimos, respetuosos de la Constitución, la ley y los Derechos Humanos, con una férrea vocación de servicio y abnegación para ganar la guerra. (EsACE, s. f.)

En la Esace se realizan los cursos de Comando (ascenso de capitán a mayor), Intermedio (ascenso de teniente a capitán), Capacitación Avanzada (CAPAVAN) (ascenso de sargento segundo a sargento viceprimero), Liderazgo Sargento Mayor de Comando y Preparador de Instructores (CPI) y Curso Exámenes de Comprobación de Asenso Sargento Primero a Sargento Mayor. Todos ellos de manera presencial.

\section{Instrumento}

La técnica de recolección de datos fue una encuesta voluntaria y en línea que se aplicó a los estudiantes. El número de participantes que contestaron el cuestionario fue de 294, de un universo de 1.200 estudiantes militares que realizaban sus respectivos cursos de ascenso en el semestre. Entre los participantes se encontraban oficiales y suboficiales del Ejército Nacional de Colombia.

Se empleó como instrumento un cuestionario de cincuenta preguntas, de las cuales 47 eran de respuesta cerrada y 3 de respuesta abierta. Estas fueron 
divididas por categorías con el fin de evaluar aspectos relacionados con la información demográfica del encuestado, al igual que conexión, pedagogía, satisfacción, aprendizaje, diseño tecnológico, gestión y orientación, que arrojaron resultados para evaluar la dimensión tecnológica.

Con las preguntas abiertas se quiso brindar la opción de recopilar la experiencia de los estudiantes de manera cualitativa con respecto a inconformidades y satisfacciones de la experiencia de aprendizaje virtual durante el tiempo del confinamiento como consecuencia de la pandemia de la Covid-19.

Algunas de las preguntas cerradas se midieron con una escala tipo Likert con cinco opciones de respuestas, que van desde "1" para totalmente en desacuerdo hasta "5" para totalmente de acuerdo. En el resto, solamente se tabularon las respuestas, organizándolas de mayor a menor rango de respuestas comunes.

De igual manera, se tuvieron en cuenta los comentarios, las observaciones y las recomendaciones que varios profesores, especialmente los que dictan la materia de Expresión Oral y Escrita, dieron a conocer en las reuniones previas y finales de sus cursos, como una acción de mejora y control a los coordinadores de materia y cursos en la EsACE. También se utilizó la experiencia docente de las autoras.

\section{Resultados}

La encuesta inicial evidencia que solamente el 95,6 \% de los encuestados aceptó participar en este estudio, mientras que el 4,4 \% que marcó la opción de "no participar", respondió finalmente las preguntas. De lo cual se deduce que existía una motivación por dar su aporte a esta investigación.

Con referencia a la información demográfica, todos los encuestados fueron militares activos: oficiales $(55,1 \%)$ y suboficiales $(44,9 \%)$, la gran mayoría $(85,7 \%)$ con un rango de edad entre 31 y 40 ańos y casi todos de género masculino (98,6\%).

Los encuestados realizaron de manera virtual cursos de Capavan (43,2\%), Intermedio (40, $8 \%$ ), Comando (13,9\%), Curso Exámenes de Comprobación de Asenso Sargento Primero a Sargento Mayor (1,7\%) y Curso Liderazgo Sargento Mayor de Comando (0,3 \%). 
El 75,2\% de los encuestados realizaron sus cursos virtuales en su residencia, el 21,8 \% en su lugar de trabajo y solamente el 2,7 \% estuvo en zona de orden público del país. En su mayoría se encontraban ubicados en la ciudad de Bogotá, D. C.; sin embargo, se registró un número considerable de estudiantes en los departamentos de Cundinamarca (12,5\%), Tolima (8,5\%), Antioquia (7,1\%), Cauca (4,7\%), Cesar (4,7\%) y Huila (4,7 \%).

No hubo alumnos en San Andrés y Providencia, Amazonas, Caldas, Guainía, Sucre, Vaupés ni Vichada, y solo el 11,6 \% de los encuestados estaban en zona rural.

\section{Dimensión tecnológica}

De acuerdo con los resultados cuantitativos, la evaluación de la dimensión tecnológica fue buena en los criterios y elementos de medición, teniendo en cuenta que la encuesta no arrojó datos en falencias de hardware, software e internet como elementos necesarios para poder tener conexión y conectividad al momento de realizar el proceso de aprendizaje virtual (tabla 1).

Tabla 1. Porcentajes de evaluación de la dimensión tecnológica

\begin{tabular}{lcc}
\hline Criterios & Medición & Porcentaje obtenido \\
\hline & Blackboard & $100 \%$ \\
\cline { 2 - 3 } $\begin{array}{l}\text { Funcionabilidad y } \\
\text { disponibilidad del } \\
\text { software }\end{array}$ & Learn & $100 \%$ \\
\cline { 2 - 3 } & Cedoc 36o & $100 \%$ \\
\cline { 2 - 3 } & $\begin{array}{c}\text { Redes sociales (WhatsApp) } \\
\text { Correo electrónico }\end{array}$ & $100 \%$ \\
\hline \multirow{2}{*}{$\begin{array}{l}\text { Funcionalidad y } \\
\text { disponibilidad del } \\
\text { hardware }\end{array}$} & Computador de mesa & $100 \%$ \\
\cline { 2 - 3 } & Portátil & $10,5 \%$ \\
\cline { 2 - 3 } & Celular & $87,8 \%$ \\
\hline \multirow{2}{*}{\begin{tabular}{cc} 
Calidad del internet \\
\cline { 2 - 3 }
\end{tabular}} & Equipo compartido & $1,4 \%$ \\
\cline { 2 - 3 } & Wifi & $29,6 \%$ \\
\hline
\end{tabular}

Fuente: elaboración propia. 
Teniendo en cuenta la experiencia que tuvieron los estudiantes, tanto en la dimensión organizativa como en la tecnológica, los resultados cualitativos arrojaron falencias con respecto al servicio de internet - que en muchas ocasiones era inestable - y la plataforma, la cual no es amigable ni agradable para los estudiantes. Relacionado con estos aspectos, se presentan tres testimonios: "La plataforma utilizada por la EsACE presenta dificultades para mantener las clases" (cuestionario 12); "me preocupa que por las limitaciones de internet o las herramientas informáticas se pueda ver afectada nuestra carrera militar en cuanto a preparación y formación" (cuestionario 193) y "en algunas horas del día la señal de internet se satura y no se entiende en algunos momentos lo que explica el docente" (cuestionario 280).

Por otra parte, los estudiantes se sintieron satisfechos por la manera como el Ejército Nacional, a través de la EsACE, continuó con su formación en línea, lo cual demuestra el interés en apoyarlos con la continuidad de su carrera, ya que "fácilmente la institución ha podido detener el proceso de capacitación y retardar a los militares, lo cual nos afectaría para los ascensos", señaló uno de los suboficiales encuestados que realizó el curso de CaPAVAN de manera virtual.

Esto también se corrobora en la encuesta, pues 166 estudiantes manifiestan estar muy satisfechos con la forma como la EsACE ha respondido ante la crisis de pandemia para la continuidad de sus estudios y otros 121 se sienten satisfechos. Solamente 3 personas se manifestaron insatisfechas ante la situación y a 4 les es indiferente el asunto.

En cuanto a la interfaz de Blackboard, esta herramienta ha sido bien aceptada por los alumnos. De los encuestados, 145 se sienten muy satisfechos, 115 satisfechos y solo a 9 les es indiferente esta herramienta. El grado de insatisfacción fue menor: 4 muy insatisfechos y 21 insatisfechos.

\section{Dimensión organizativa}

Teniendo en cuenta que la dimensión organizativa es ajena a los estudiantes, pero no a las instituciones educativas, las conclusiones propias, obtenidas al momento de realizar la investigación, o aquellas que pudieron nutrirla a través de charlas informales y cotidianas con los docentes de la EsACE, evidencian que el personal de profesores y del área administrativa manifestaron en varias ocasiones que el proceso de enseñanza-aprendizaje no se debilitó en tiempos de pandemia gracias al apoyo del gobierno nacional y a que el mando 
del Ejército Nacional decidió continuar con la programación educativa para los miembros de la Fuerza.

El sistema educativo militar se amplió, se implementaron opciones de cambio y se acogieron las mismas estructuras de presentación, conservando la mística y el perfil que siempre ha implementado la EsaCE. Así, tanto civiles como militares fueron partícipes de la transición de la educación presencial a la virtual y en línea, siempre con la disciplina, la práctica y el liderazgo como ejes transversales de la formación.

La institución se esforzó y gracias al trabajo en equipo, la preparación y el fortalecimiento de los recursos, tanto humanos como tecnológicos, previamente contratados y adquiridos, realizó un montaje acelerado de las clases virtuales, capacitó a los docentes e instructores para continuar con el liderazgo de la educación reglamentaria para su personal de oficiales y suboficiales.

La transformación de lo presencial a lo virtual en la ESACE también deja tareas por hacer: actualizar las plataformas digitales, capacitar previamente a los estudiantes sobre el uso de programas ofimáticos y hacer cambios en la forma de dirigir la política educativa a través de las Tic para continuar con la formación y el aprendizaje basados en la filosofía y ética del militar. Seguramente será una tarea fácil, considerando que la institución cuenta con los componentes necesarios en la modalidad presencial, de tal modo que solo resta adaptarlos a las nuevas tecnologías (figura 2).

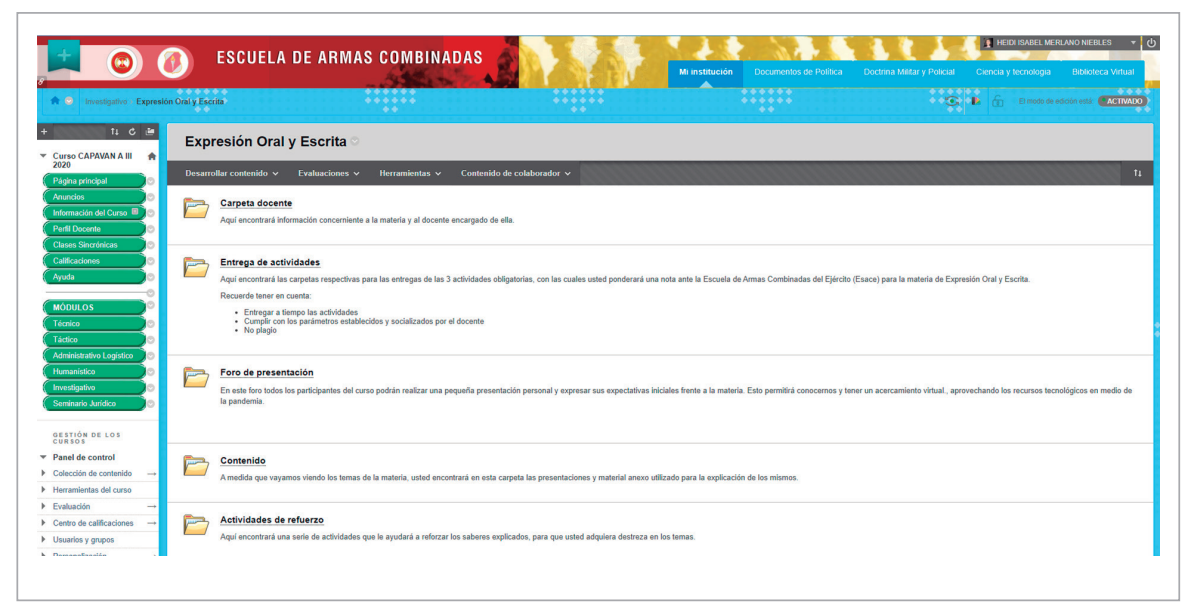

Figura 2. Diseño del curso de Expresión Oral y Escrita en Blackboard.

Fuente: elaboración propia. 


\section{Dimensión pedagógica}

El estudio cuantitativo arrojó que el 62,2\% de los estudiantes encuestados manifestaron que las herramientas pedagógicas utilizadas en el curso siempre fueron totalmente complementarias al saber de las materias, mientras que el 30,3\% dijo que casi siempre. Así, solamente 14 militares eligieron la opción "a veces", 5 "casi nunca" y 3 "nunca".

Además, 243 encuestados consideraron que las estrategias didácticas que implementaron los docentes en cada una de las materias durante este aprendizaje en línea fueron totalmente adecuadas. Solamente 37 estudiantes manifestaron que en algunas materias fue evidente esta planificación del proceso de enseñanza-aprendizaje y 14 afirmaron no haber percibido ninguna.

En lo concerniente a las evaluaciones por parte de los docentes, más de la mitad de los encuestados manifestaron que siempre fueron apropiadas (161 encuestados), el 32,7\% dijo que casi siempre lo eran, 27 estudiantes se mostraron indiferentes ante la cuestión planteada y 10 estuvieron en desacuerdo: 5 dijeron que nunca y los otros 5 que casi nunca.

Con base en lo anterior, se puede deducir que los estudiantes aprueban los objetivos de funcionamiento de los cursos y que estos se han cumplido de manera conforme y satisfactoria.

En referencia con el estudio cualitativo, los estudiantes manifiestan que los objetivos de las materias se están cumpliendo, ya que están muy relacionados con los saberes y que estos pueden ser bien aplicados en el futuro cercano en sus carreras militares. De otro lado, los comentarios negativos van dirigidos a que las actividades son muy densas, lo cual "dificulta su realización oportuna y en ocasiones requieren tanta atención que hasta hay que dejar de atender a otras materias, teniendo en cuenta que los horarios son muy largos y ocupan casi todo el día" (cuestionario 135).

De igual manera, los estudiantes señalan que los contenidos de los saberes son buenos y prácticos para su carrera militar, lo que da relevancia a los cursos que realizan en la EsACE. Sin embargo, las sugerencias y recomendaciones que entregaron los alumnos evidencian que prefieren clases dinámicas y activas, en vez de charlas constantes, lo cual se puede convertir en una deficiencia en la educación en línea. 
Asimismo, los encuestados perciben con claridad y lógica el contenido de sus cursos, pero mencionan que prefieren no realizar actividades de trabajo en grupo en la modalidad en línea porque se les dificulta coordinar, comunicarse y poner en marcha las tareas, ya que muchos se escudan, en algunas ocasiones, en las fallas de internet y los cortes de energía eléctrica para no realizar aportes $\mathrm{y}$ actividades colaborativas.

Así las cosas, resulta evidente que la interactividad es un aspecto poco relevante para los estudiantes, teniendo en cuenta que ellos le dan mayor importancia a ese aspecto solamente con el docente, pero no con sus compañeros de clase ni con los comandantes de curso. De hecho, en este valor los porcentajes fueron bajos y muy repartidos, siendo la valoración igual o menos que antes de las que lidera la encuesta en aspectos relacionados con la cantidad de interacciones con los docentes $(31,6 \%-22,8 \%)$, con el personal militar $(28,3 \%-22,2 \%)$ y con los compañeros $(20,7 \%-30,3 \%)$.

\section{Dimensión de gestión}

El conjunto de servicios que reciben los estudiantes de manera previa y durante al curso fue la dimensión mejor valorada. La orientación, el acompañamiento, el seguimiento, la motivación y el asesoramiento fueron los criterios más sobresalientes tanto en la medición cuantitativa como en la cualitativa.

$\mathrm{El}$ acceso oportuno y la orientación previa al inicio de los cursos fue un factor que resaltaron los alumnos cuando expresaron su grado de satisfacción con el docente al finalizar sus materias. Sin embargo, en las reuniones que sostuvieron los docentes entre ellos, con los estudiantes, o con los directores de materias han manifestado que los alumnos deben tener conocimientos y habilidades previas en temas relacionados con el manejo de plataformas y uso de herramientas de ofimática y de la web, para que puedan afianzar sus conocimientos y desempeñarse de manera óptima durante el desarrollo de los cursos.

Una de las docentes, de la materia de Expresión Oral y Escrita de la ESACE, manifiesta que en ocasiones tuvo que dedicar tiempo de la clase para hacer seguimiento, acompañamiento y esclarecer el buen uso de la plataforma Blackboard Learn, así como para recordarles a los estudiantes cómo cargar las actividades, los exámenes y los foros en esta herramienta virtual, en lugar de destinarlo para explicar los saberes y desarrollar las actividades en línea. 
Razones como esta han hecho que la orientación y el acompañamiento que hacen los docentes a los estudiantes se hayan afianzado más que en el proceso de aprendizaje presencial. La atención ha sido oportuna y eficaz, lo cual se puede deducir por los reportes de notas y aprobaciones de las materias.

\section{Discusión}

Los resultados que se presentaron en la sección anterior muestran las percepciones de un grupo de alumnos militares sobre los cursos de ascenso en el año 2020, los cuales se realizaron en línea ante la crisis ocasionada por la pandemia de Covid-19.

Las percepciones de los estudiantes evidencian diferencias significativas en las cinco dimensiones consideradas en la encuesta (pedagógica, tecnológica, de gestión, diseño e interfaz y de orientación) y dan cuenta de que la nueva modalidad obtuvo una buena aceptación entre los alumnos, aunque con algunas dificultades. Es decir, parece que tuvo un impacto positivo el hecho de que se haya incorporado una instancia virtual en la que pudieran participar desde su residencia o trabajo en la plataforma de formación, ya que esto les permitió continuar con su curso de ascenso. Sin embargo, esta modalidad hizo que los estudiantes tuvieran que dedicar más tiempo a las actividades académicas y, en algunos casos, desarrollarlas simultáneamente con su trabajo o quehaceres de apoyo en el hogar.

Una educación virtual de calidad debe cumplir ciertos requerimientos mínimos, tanto técnicos como metodológicos, y responder a las necesidades de los alumnos, a quienes se les debe proporcionar un material didáctico apropiado que les aporte conocimiento durante las clases. Al respecto, Alba Lucía Sánchez (2005), en su artículo “¿Cómo deben ser los contenidos para un curso virtual?”, señala que deben tomarse en cuenta los procesos relacionados con la gestión de los contenidos en el diseño de los materiales, la coherencia didáctica, las ayudas para el estudio, las actividades, los recursos académicos consistentes y el uso de las herramientas de comunicación sincrónicas y asincrónicas.

En cuanto a la dimensión pedagógica, es evidente que el papel del docente como agente motivador y como guía en el proceso de construcción del conocimiento es fundamental, pero este papel no puede ser opacado por la falta de implementación de didácticas. Por ello, se recomienda que la EsACE 
realice constantemente un instructivo o capacite a su personal docente, tanto civil como militar, sobre actividades de interacción, las cuales son muy valiosas y oportunas al momento de desarrollar un saber, bien sea a través de la virtualidad o de manera presencial.

Asimismo, en cuanto a las herramientas de enseñanza, los alumnos reportaron que están satisfechos de que se haya vinculado la plataforma Blackboard, la cual permitió el desarrollo de las clases, y de que los docentes hayan adaptado su metodología y herramientas al nuevo escenario. Sin embargo, la necesidad constante de contar con una buena conexión de internet para que esta herramienta funcione a la perfección hizo que esa complacencia cambiara.

Los constantes inconvenientes en la comunicación, la conectividad y al cargar las actividades hicieron que los estudiantes estuvieran inconformes con la plataforma Blackboard durante el 2020, año en el cual la educación a través de la virtualidad fue esencial para que avanzaran en sus procesos formativos, que son un requisito para que puedan ascender de un grado militar a otro superior.

Es importante decir que la educación virtual y en línea se fundamenta en el aprendizaje, a diferencia de la presencial, en la cual tiene más importancia la enseñanza, de tal modo que el reto no consiste en pasar lo presencial a un aula virtual, sino en generar materiales que respondan a las nuevas dinámicas educativas.

La calidad del internet en algunas regiones, especialmente las rurales, fue otro factor que se detectó como falencia; situación adversa para la implementación de la educación virtual en la EsACE, pero esencial para el buen desarrollo de aprendizaje en los estudiantes. Sus inconvenientes se salen de las manos de la institución. Muchas de las fallas en internet se deben a la cobertura de banda ancha que posee Colombia en su territorio nacional.

Frente a este panorama, la ESACE, como institución encargada de la formación de los militares, se acogió a las medidas preventivas en salud establecidas por el gobierno de Colombia, que generaron nuevas dinámicas en la enseñanza de los cursos que imparte y ante las cuales la Escuela tuvo respuestas exitosas con su programación anual. Sin embargo, se presentan desafíos para la mejora en el campo virtual, como la actualización de las herramientas tecnológicas y las metodologías pedagógicas pertinentes para la educación virtual por parte de los docentes. Esto demanda el uso de tecnologías de simulación, 
gamificación y recursos de realidad mixta que la posicionen como una institución a la vanguardia en la actualidad.

Con respecto a los resultados cualitativos sobre gestión y orientación, los puntajes más altos con base en las puntuaciones de los estudiantes fueron obtenidos por el valor de entrega, la dedicación y el apoyo que recibieron de sus superiores, la institución y sus docentes, al igual que la actitud que estos tuvieron durante el proceso de aprendizaje, que para muchos fue nuevo.

\section{Conclusión}

Este estudio cuantitativo y cualitativo sobre la experiencia de enseñanza virtual en los cursos de formación militar en la EsACE nos hace reflexionar sobre un proceso de educación que cumple con las dimensiones del aprendizaje propuestas por científicos y estudiosos del tema a nivel nacional e internacional.

Por lo tanto, se puede concluir que los alumnos militares valoran el esfuerzo que ha hecho la EsACE para permitir la continuidad de los cursos de ascenso usando la plataforma Blackboard. Sin embargo, se hace necesario que este espacio sea actualizado y posea una interfaz más atractiva y versátil para que sea más eficiente su uso.

La plataforma Blackboard es una herramienta al servicio de los docentes y los alumnos que permite desarrollar aprendizajes más dinámicos e innovadores, por ello es necesario hacer capacitaciones para que el personal mejore las metodologías didácticas en esta modalidad virtual. En este sentido, es de suma importancia incluir herramientas tecnológicas que faciliten la interacción digital.

Asimismo, se destaca la capacidad orientadora de los docentes, así como su acompañamiento en los espacios sincrónicos y asincrónicos del curso. No obstante, teniendo en cuenta la experiencia del personal docente y de los estudiantes en el manejo de la plataforma y los medios tecnológicos de la institución y los propios, el análisis de la encuesta evidencia que es necesario actualizar los conocimientos en el manejo de herramientas virtuales de aprendizaje y competencias digitales, especialmente por parte de los docentes, con lo cual se estaría velando por la calidad de la enseñanza, pues estas aportarían a la construcción de metodologías didácticas innovadoras. 
Aunque tuvieron algunas dificultades, cabe resaltar que los alumnos reportaron satisfacción personal con respecto al apoyo institucional, de los compañeros y de sus familias para poder terminar su curso en línea en medio de la pandemia de la Covid-19.

De forma general, los resultados cuantitativos de este estudio muestran que las dimensiones del modelo fueron evaluadas, en promedio, de modo aceptable, pero que desde la perspectiva del alumno hay algunos criterios que es urgente mejorar, como la disponibilidad de la plataforma, la calidad en el servicio de internet y la interactividad entre alumno y docente.

\section{Referencias}

Blanchard, M., \& Muzas, M. (2016). Los proyectos de aprendizaje. Un marco metodológico clave para la innovación. Narcea Ediciones.

Bournissen, J. (2014). Modelo pedagógico virtual. Signos Universitarios, 1(2), 245-266. http:// p3.usal.edu.ar/index.php/signos/article/view/2113

Caracol Radio. (2019, febrero 5). 20 millones de colombianos no cuentan con banda ancha: MINTIC. Caracol Radio. https://cutt.ly/Xf3NkdN

Casanova, M. (1992). La evaluación, garantía de calidad para el centro educativo. Edelvives.

Castellanos, Á. (2014). Pedagogía y calidad educativa en la era digital y global. Ecoe Ediciones.

Escuela de Armas Combinadas del Ejército [ESACE]. (s. f.). Reseña histórica. https://www. esace.mil.co/escuela_armas_servicios/conozcanos_401261

González, M. (2000). Evaluación del aprendizaje en la enseñanza universitaria. Revista Pedagogía Universitaria, 5(2), 31-61.

Khan, B. H. (2001). Web-based training. Educational Technology Publications.

Salinas, J., Negre Bennasar, G., \& Escandell. (2006). Modelos didácticos en entornos virtuales de formación: identificación y valoración de elementos y relaciones en los diferentes niveles de gestión. Congreso Internacional EduTEc'06: La educación en entornos virtuales: calidad y efectividad en el e-learning, Tarragona, España.

Sánchez Alzate, A. L. (2005). Apuntes sobre: ¿Cómo deben ser los contenidos para un curso virtual? Revista Virtual Universidad Católica del Norte, (14). https://revistavirtual. ucn.edu.co/index.php/RevistaUCN/article/view/262/496

Talavera, N. (2011). La formación en línea: perspectivas organizacional, pedagógica, tecnológica y socio-cultural. http://upcommons.upc.Edu/e-prints/bitstream/2117/15588/1/ DTI_La\%20formaci\%C3\%B3n\%20en\%201\%C3\%ADnea.\%20Perspectivas\%20 organizacional, $\% 20$ pedag $\%$ C3\%B3gica, $\% 20$ tecnol\%C3\%B3gica $\% 20 y \% 20$ sociocultural.pdf

Tenbrink, T. D. (2006). Evaluación: Guia práctica para profesores (8a ed.). Nacea Ediciones. 